\title{
Identifying Barriers to Women's Participation in Sports Activities in both Urban and Rural Communities
}

\author{
Somayeh Farzaneh ${ }^{1}$, Rosa Rahavi Ezabadi ${ }^{1, *}$, Samaneh Sadat Khalili Rad ${ }^{2}$, Pari Khalili Marandi ${ }^{3}$, \\ Vikram Ranawat ${ }^{4}$ \\ ${ }^{1}$ Department of Motor Behaviour, Faculty of Sport Sciences, Alzahra University, Tehran, Iran \\ ${ }^{2}$ Department of Physical Education, Faculty of Humanities and Social Sciences, Yazd University, Yazd, Iran \\ ${ }^{3}$ Education Faculty, University Kebangsaan Malaysia (UKM), Malaysia \\ ${ }^{4}$ Faculty of Sciences, University of Rajasthan, India
}

Received January 27, 2021; Revised April 7, 2021; Accepted May 10, 2021

\section{Cite This Paper in the following Citation Styles}

(a): [1] Somayeh Farzaneh, Rosa Rahavi Ezabadi, Samaneh Sadat Khalili Rad, Pari Khalili Marandi, Vikram Ranawat, "Identifying Barriers to Women's Participation in Sports Activities in both Urban and Rural Communities," International Journal of Human Movement and Sports Sciences, Vol. 9, No. 3, pp. 536 - 542, 2021. DOI: 10.13189/saj.2021.090320.

(b): Somayeh Farzaneh, Rosa Rahavi Ezabadi, Samaneh Sadat Khalili Rad, Pari Khalili Marandi, Vikram Ranawat (2021). Identifying Barriers to Women's Participation in Sports Activities in both Urban and Rural Communities. International Journal of Human Movement and Sports Sciences, 9(3), 536 - 542. DOI: 10.13189/saj.2021.090320.

Copyright $(2021$ by authors, all rights reserved. Authors agree that this article remains permanently open access under the terms of the Creative Commons Attribution License 4.0 International License

\begin{abstract}
Today, the role of women in social activities in Iranian society is more important than ever. Despite the increasing presence of women in the social arena, their participation and attendance in sports activities are limited. The purpose of this study is to investigate the barriers affecting women's non-participation in sports activities in both urban and rural communities. The data of this study were collected using a questionnaire on barriers to women's participation in sports activities. Findings indicated that the two groups of urban and rural women reported similar priorities, with the highest priority being related to economic and personal barriers. The lowest priorities were related to family, cultural barriers. With extensive and targeted advertising, a variety of mass entertainment programs of sports activities can be assigned to the adult age categories. Such interventions will lead to better welfare and quality of life for women in urban and rural communities.
\end{abstract}

Keywords Barriers, Rural Society, Sports Activities, Urban Society, Women

\section{Introduction}

Physical activity is one of the main areas of health and an important lever in providing a desirable lifestyle and better quality of life. These two categories are inextricably linked, and the institutionalization of desirable health and social behaviors depends on the lifestyle of individuals in each society Rahavi Ezabadi [1]. So far women has made up half of the human resources in the development of societies, socio-economic and cultural development programs. Thus, the way should be paved for better use of their abilities and talents. Thus, their effective presence ensured in the process of sustainable development in various social fields and the ground should be provided for their effective participation in cultural development Barqarar [2]. Also, according to social psychologists and experts, a healthy society is based on healthy women and mothers are the main pillars of the family. Therefore, they must be in perfect health. On the other hand, the health of each member of society, especially women, directly affects the overall health of the family Zahirinia [3]. In order for all members of society to have a healthy body and not suffer from mobility, inactivity, and disability with age, it is necessary to have an effective and desirable physical fitness at the initial level in different stages of life Safari Shali [4]. This by far is due to the increasing prevalence of mental health issues/disorders in society, especially among women. It may also be due to a greater sense of responsibility towards family members under high psychological stress and exposure to mental and physical 
harm Khodadadi [5]. According to statistics published by the Centre for Women's Participation, girls and women make up more than half of the country's population. Unfortunately, by the beginning of the third millennium, the percentage of women in sports activities in different countries is still different (or lower than men) Ehsani [6]. In all countries, women are less than half of men and boys actively involved in sports. In Iran, girls and women face a serious problem of inactivity due to the existence of various barriers to the use of sports facilities. Sports participation in addition to physical fitness, will strengthen morale, reduce group conflicts, as well as improve the quality of life and the development of sports talents Beirami [7].

Rural and nomadic women make up about 10 million people or $14 \%$ of the country's population and $49 \%$ of the rural and nomadic population Moeidfar [8]. Some women, in addition to doing housework, along with men, engage in physical activity and other routine activities of rural life. Sometimes, the busy schedules of rural women and some other factors (cultural, economic, etc.) do not allow them to have much fun and entertainment. To improve the quality of life for this part of the population, it is necessary to give more attention and thinking needed to improve mental and physical health Farzizadeh [9]. Despite the importance of the role of women in Iran, little attention is given with regard to position of women as an important and influential part in the development of society Rostami [10]. Rural women confront issues of being overworked on the farm and at home, accessibility issues to recreation centers. Saadi [11], feeling insecure, poor communication skills, illiteracy and low literacy, high working hours Chen [12], doing more agricultural activities, deprivation of educational services, physical weakness and also low level of health Chiou[13], These and other cases, significantly reduce the quality of life, especially health-related quality. A study examined the situation of women's leisure in rural society and the results showed that rural women of the young generation due to changing values are not very interested in doing agricultural activities. While, compared to the middle-aged and older, more leisure time has been created for them Farzizadeh [9].

Results of the findings of $[14,15]$, regarding the barriers for rural and urban women in physical activity, showed that rural women are less active than urban women and more prone to obesity. It is reported that cultural and economic barriers to be the most important barriers for women in physical activity. Effective care interventions to promote physical activity should address barriers and motivating factors in these three categories of determinants for maximum efficacy.

Results of another study that examined and analysed the issues and problems affecting women's participation in sports activities indicated five barriers related to social, cultural, personal, family and economics, which has led to a decrease in participation of women in sports $[16,17]$.
Compared the urban and rural communities of India, it can be found that there was no difference in the two communities in terms of physical activity, such as work, transportation and recreation. However, it was found that rural women do $13 \%$ more physical activity than urban women. Therefore, it is necessary for the decision-makers and the authorities to provide the necessary conditions and greater and more active presence of women in the field of sports. In this regard, it is necessary to identify the issues and problems in this way through initial studies it is vital to support this recommendation with the current evidence and research studies. By participating in sports activities, it changes women's perception of themselves and the richness of their experiences. Today, women's exercise is one of the main topics in the physical fitness movement to take advantage of the benefits of physical fitness, especially the feeling of well-being and achieve goodness throughout life. Therefore, this study aims to identify and prioritize the barriers affecting the presence and participation of Iranian women in physical activity so that it can use the necessary strategies to eliminate these barriers in both urban and rural communities. Therefore, according to the mentioned cases and also reviewing the researches done in this regard, this research seeks to answer these questions: 1- What are the main obstacles facing women in Khorasan Razavi province in both urban and rural communities? 2. What are the priorities of each of these obstacles?

\section{Research Method}

The Present study is practical.In terms of descriptive method, it is of correlation type. The statistical population of this study was women living in Koosheh village of Kashmar city of Khorasan Razavi province as a rural community and also women living in Mashhad city of Khorasan Razavi as an urban community. In the rural community, questionnaire on barriers to women's participation in sports activities that had content, formality and criteria validity.

Questionnaires were delivered to 150 rural women who filled out 110 questionnaires and were collected. In urban society, 150 questionnaires were provided for participants online and urban women answered 107 questionnaires.

\section{Measuring Tools}

A questionnaire on barriers to women's participation in sports activities has been designed and developed to assess barriers to women's participation in sports activities Mirghafouri [16] This questionnaire has 30 questions and 5 dimensions (social, personal, cultural, family, economic barriers), measuring the barriers to women's participation in sports activities based on the five-choice range of Likert. This questionnaire was evaluated in terms of content 
validity, form and appropriate criteria and the reliability of the questionnaire was estimated to be 0.85 through the alpha coefficient of Cronbach, which indicates the reliability of the questionnaire.

\section{Data Analysis Methods}

In order to analyse the results of the collected data at the level of descriptive statistics, the mean, and standard deviation for descriptive information and in the inferential statistics section for data analysis, statistical methods of Kolmogorov-Smirnov test were used for normal data. There was no normal distribution of data. Therefore, the non-parametric Friedman test was used to analyse the data. All statistical operations were performed using SPSS software version 16 . The significance level was considered 0.05 .

\section{Findings}

Analysis of demographic data of participants showed that the mean age of urban women was 30.9277 .45 and rural women were 29.5488 .32 (Table 1).

Table 1. Mean and standard deviation of age group

\begin{tabular}{|c|c|c|}
\hline Group & Mean & Std \\
\hline Urban & 30.92 & 7.45 \\
\hline Rural & 29.54 & 8.32 \\
\hline
\end{tabular}

Findings related to marital status showed that out of 108 people in the urban group, $26 \%$ were single and $74 \%$ were married; and out of 110 people in the rural group, $11 \%$ were single and $89 \%$ were married (Table 2).

Table 2. Frequency of participants based on marital status

\begin{tabular}{|c|c|c|c|}
\hline Group & Statues & Frequency & Percentage \\
\hline \multirow{3}{*}{ Urban } & Single & 28 & $26 \%$ \\
\cline { 2 - 4 } & Married & 79 & $74 \%$ \\
\cline { 2 - 4 } & Total & 108 & $100 \%$ \\
\hline \multirow{3}{*}{ Rural } & Single & 12 & $11 \%$ \\
\cline { 2 - 4 } & Married & 98 & $89 \%$ \\
\cline { 2 - 4 } & Total & 110 & $100 \%$ \\
\hline
\end{tabular}

Table 3. Frequency of group participants based on employment

\begin{tabular}{|c|c|c|c|c|}
\hline Variables & \multicolumn{2}{|c|}{ Urban Group } & \multicolumn{2}{c|}{ Rural Group } \\
\hline Occupation & Frequency & Percentage & Frequency & Percentage \\
\hline Housewife & 77 & $72 \%$ & 91 & $83 \%$ \\
\hline Employed & 30 & $28 \%$ & 19 & $17 \%$ \\
\hline Total & 107 & $100 \%$ & 110 & $100 \%$ \\
\hline
\end{tabular}

Findings related to the participants' occupations also showed that in the urban group, $72 \%$ were housewives and
$28 \%$ employed and in the rural group, $83 \%$ were housewives and $17 \%$ were employed (Table 3 ).

Findings related to the level of education of the participants showed that the highest level of education in the urban group was related to diploma $51 \%$ and the lowest level of primary education was $0 \%$. For the rural group the highest level of education was related to the secondary of $40 \%$ and the lowest level of master and doctoral education $0 \%$ (Table 4 ).

Table 4. Frequency of group participants based on education

\begin{tabular}{|c|c|c|c|c|}
\hline Variables & \multicolumn{2}{|c|}{ Urban Group } & \multicolumn{2}{c|}{ Rural Group } \\
\hline Education & Frequency & Percentage & Frequency & Percentage \\
\hline Primary & 0 & $0 \%$ & 42 & $38 \%$ \\
\hline Secondary & 5 & $5 \%$ & 44 & $40 \%$ \\
\hline Diploma & 54 & $51 \%$ & 19 & $17 \%$ \\
\hline Associate & 10 & $9 \%$ & 1 & $1 \%$ \\
\hline Bachelor & 29 & $27 \%$ & 4 & $4 \%$ \\
\hline Master & 8 & $7 \%$ & 0 & $0 \%$ \\
\hline Doctoral & 1 & $1 \%$ & 0 & $0 \%$ \\
\hline Total & 107 & $100 \%$ & 110 & $100 \%$ \\
\hline
\end{tabular}

In the inferential section, the normality of data distribution was first examined using the Kolmogorov-Smirnov test, which showed that the data distribution was not normal $(\mathrm{P} \geq 0.05)$. Therefore, non-parametric Friedman statistics were used for the inferential analysis of research data. The results of the Friedman ranking test showed that for both urban and rural women, there is a significant difference between the barriers to participation in physical activity in terms of importance.

Table 5. Results of Friedman test for ranking barriers to physical activity participation of urban and rural women

\begin{tabular}{|c|c|c|c|c|}
\hline Group & Frequency & df & K Square & Sig \\
\hline Urban & 107 & 4 & 266.44 & 0.0001 \\
\hline Rural & 110 & 4 & 264.45 & 0.0001 \\
\hline
\end{tabular}

*It is significant at the level of $\mathrm{p} \leq 0.05$

Table 6. Mean rank and priority of barriers to participation in physical activity of urban and rural women

\begin{tabular}{|c|c|c|c|c|}
\hline Variables & \multicolumn{2}{|c|}{ Urban Group } & \multicolumn{2}{c|}{ Rural Group } \\
\hline Status & Mean rate & Priority & Mean rate & Priority \\
\hline Economic & 4.27 & 1 & 4.29 & 1 \\
\hline Private & 4.13 & 2 & 4.26 & 2 \\
\hline Social & 3.12 & 3 & 2.26 & 3 \\
\hline Cultural & 1.91 & 4 & 2.24 & 4 \\
\hline Family & 1.58 & 5 & 1.95 & 5 \\
\hline
\end{tabular}

Comparison of the priorities of barriers to participation in physical activity showed that both urban and rural 
women reported similar priorities in terms of average rank, so that the highest priority was related to economic barriers (27.4 mean rank of urban women and 29.4 is the mean rank of rural women) and the lowest priority is related to family barriers (58.1 mean rank of urban women and 95.1 mean rank of rural women) (Table 6).

\section{Discussion}

Exercise and recreation are desirable activities that have functions such as health, vitality, promoting a dynamic lifestyle and well-being. Therefore, promoting health programs and a healthy living pattern requires the creation of facilities and removal of barriers to access to the place or places of physical activity to provide opportunities for people to participate in physical activity and people from inactivity and risk of various diseases. Be liberated physically and mentally Deborah [18]. Therefore, to encourage and maintain participation in disadvantaged populations, despite the quality of programs, availability, low cost and proximity to the place of sports can lead to regular participation in sports activities. Sport, like other phenomena in society, is influenced by social and cultural factors, and some researchers consider sport as a common cultural thing and language for global communication Laker [19]. The aim of the present study was to identify and prioritize barriers to participation in women's physical activities in both rural and urban communities. The results indicated that the priority of barriers to participation in women's physical activity in both urban and rural groups was economic barriers, personal, social, cultural and family.

Some of the most important social barriers are lack of attention of officials to women's sports, lack of support for educational canter's such as universities on sports issues, lack of successful models of women's sports as a result of research [20_24].

Social learning according to Bandura's theory places great emphasis on the role of social environment factors in learning attitudes and behaviors, although the learning process takes place in two physical environments (material aspects of the field of behavior) and social environment (real or imagined presence of others) or their participation in learning. However, the social environment seems to be of particular importance Riahi [25]. There are negative attitudes toward women's sports activities in Iranian society. Existence of some subcultures, stereotypes and family prejudices are among these problems. In a national research project conducted in 2001 entitled the position of sports in Iranian women's life among urban women aged 20 to 65 years. The participants were from 176 cities of the country. The result indicated that $45.8 \%$ of the women surveyed stated that they do not exercise Tondnavis [26].

Trigueros [27]. They found that social issues due to their gravity play an important role in improving the desired social behavior of people. Bruner [28] also found that social aspects by improving interpersonal relationships as well as building mutual trust and security cause to overshadow the desirable social behavior of individuals and the social environment due to its clarification can be the intention of individuals to exercise and participate in sports. Regarding cultural barriers such as male-cantered (Patriarchal society) culture the culture of patriarchy in the sports community, observance of Islamic decency, maintaining the hijab and harassment by others on the way back and forth are the most important obstacles mentioned by women [29-30]. A study conducted by Agergaard [30] on the 10 girls 13-17 years examined the role of religious culture as a barrier to Danish Muslim girls' participation in sports. The goal of this article was to show that Muslim female simultaneously practices their religion and sports.

The researcher mentioned that political interference, media coverage and research often refer to the lack of representatives of ethnic minorities, especially females, in physical activity and organized sports. In academic and public arguments, religious culture is one of the particular barriers to attending sports among Muslim females. Given that religious beliefs are one of the most important barriers, it is suggested that propaganda to clarify the minds of students and women that exercise is not contrary to religious beliefs in Islam religious, and therefore a constant emphasis on sports.

Also, the interaction between personal characteristics and environmental factors causes the perception of the environment around people in accordance with the facts and influenced by the personality aspects of individuals. This causes the environment to be considered safe or unsafe for people Foster [31]. The insecurity of a social environment causes the degree of dynamism of this environment to be lost. In other words, the tendency to perform public activities among individuals will be reduced. On the other hand, proper design of the environment and in accordance with the characteristics of individuals causes a suitable context to improve the participation of ones in various activities, including sports activities. Understanding the safety of an environment is one of the most important issues in any space Doyle [32]. In this regard, the extensive efforts of the police to secure the environment around women's sports venues have a significant impact on increasing the number of participants in physical activity. One of the personal barriers being lack of time due to the lack of interest and motivation isintroduced as the most important barriers for women participation in physical activity that is consistent with these researches [33_36].

The study conducted by Zimmermann [37] on the participation of rural women in southern illinois in physical activity participants described numerous, often conflicting, individual, social, and environmental barriers to physical activity and these barriers and facilitators vary at different stages of a woman's life. In fact, women face different barriers at every point in time, depending on their age, 
family circumstances, and the environment in which they live.

Leisure time is a part of a person's free time, not work time. Obviously, with the development of industry and technology, people's leisure time has increased. So, it is more important to spend leisure time in obtaining satisfaction, relaxation. Of course, leisure activities are defined in such a way that people in leisure time should be considered away from any personal, social, family and religious commitments Saraie [34]. Regarding the leisure time of rural women, Farzizadeh [9]. It depends on different seasons of the year, considering the amount of agricultural land, water, livestock and poultry which essentially determine the amount of leisure time of rural women. Also, the type of leisure activities and the amount of time devoted to it have changed in different generations of rural women compared to the past. Young rural women are less interested in farming due to changing values. The middle-aged generation has more leisure time compared to the older generation. Also, the type of leisure activities has changed due to new developments Saraie [34].

One way to increase sports participation among groups is to improve leisure programs. Leisure programs as an important and fundamental issue play an important role in increasing participants in the sports activity. In other words, strengthening these programs can lead to the institutionalization of sports among different groups Dagkas[38]. Evenson [39], found that environmental issues through social groups attract people to different programs. In addition, environmental factors reflect the conditions that attract people to activity. Therefore, environmental factors influence people's minds and stimulate them to participate in sports programs. Even, increasing social relationships, getting help from professionals, exercising patience and focusing on popular sports activities, can increase people's interest and desire for sports.

According to research results, in terms of family barriers, parents and spouse opposition to sports, fear of academic failure and child care and housework are the most important barriers that are consistent with these researchers Torkfar [40], Sit [36], Saraie [34]. Women's health is the foundation of family health and their participation in providing, maintaining and promoting community health is undeniable. The importance and centrality of the family are with women, and a dynamic and healthy society is achieved through a healthy family environment. According to our beliefs, the family is of special importance. Women can be the centre and promoter of health in the family and by drawing the right lifestyle pattern for the whole family (such as proper nutrition, proper physical activity, on time food) playing an important role in preventing non-communicable diseases such as cardiovascular disease and cancer Nowruzi [41]. Due to the key role of women in the family, family members (husband and children) have a duty to accompany, provide welfare facilities and devote hours of the day to meet the personal needs, and help her reach her own true position. It will be better they all provide services to women in most hours of the day, consulting organisations with venues for women in order to provide services for female employees and show successful women in sports who have also been successful in education too. It may be effective in solving this problem.

The result of the current research indicated that barriers to facilities and economy, lack of enough investment in the development of women's sports facilities, shortage and high cost of women's sports equipment and lack of access to suitable spaces and gyms for women are the most important obstacles that are consistent with these researches [15,19_21,34,42,43]; the study indicated that low-income populations, including racial/ethnic minorities and people with low socioeconomic status, adequately participate in the recommended levels of physical activity but do not match.

Always, restrictions are a barrier to any kind of leisure activity. Although the limitations must be reduced in various ways. Exercise is a reality that can and should be realized in the simplest way in the living environment of each person Saraie [34].

The result of the present study showed that women are interested in participating in low-cost sports and the high cost of equipment is a barrier to their participation in sports activities. As the lack of proper sports facilities in their place of residence is one of the most important obstacles to attending sports activities. Of course, offices cannot allocate a venue to their employees in any place, but it will be possible to cover part of the costs of people's participation in sports activities. In order to improve the situation of women in this dimension, the construction of sports facilities for women should be supported through long-term loans with low-interest rates, and women's sports centers shold be established in consultation with charities to build a women's gym. For rural women, a suitable place should be found to build gyms in the city center and areas close to villagers' homes that should be open to the public.

Therefore, according to the results of this study, despite the fact that rural women had a lower level of education and sports facilities than urban women, but in terms of barriers to participation in physical activity and sports, give almost the same results. This shows that the obstacles facing women in sports and physical activity are the same in both urban and rural communities of Khorasan Razavi province. The most important of these barriers are economic and personal barriers. Perhaps it can be said that by removing personal barriers and economic barriers, it will become easier to promote sports among the women. If every woman, value her health, freshness, beauty, she will realise value and importance of sports and physical activity. As a result, she will be more interested and try harder to remove economic and other barriers.

\section{Conclusions}

Comprehensive development of sports and participation 
in women's sport activities requires changing the attitudes of managers and policy makers in order to create infrastructure, provide more and better facilities in terms of availability, low cost, as well as creating a suitable cultural environment in society for development and maintaining physical activity for lifelong enjoyment. In order to improve their health by participating in enjoyable and content-rich physical activity programs, for the advancement and development of women's sports, attitudes towards women's presence in society should be changed and this culture should be institutionalized, which does not mean gender segregation in sports. On the other hand, the results of research in recent decades show that the tendency, interest and motivation of women in sports have significantly increased. But they still do not participate in any physical activity. In order for sports to become pervasive in their lives and to find the real place of exercise in the daily lives of girls and women in society, women's sports should be considered as one of the essential needs of society. In turn, it increased productivity and disease prevention to provide welfare and an active lifestyle as well as a healthy lifestyle model for women in the community.

\section{Acknowledgments}

Finally, the authors of this article would like to express their gratitude to the women who accompanied us in the process of this research.

\section{REFERENCES}

[1] Rahavi Ezabadi, Rosa. \& Razavi, Ameneh. (2017), The Statues of Physical Activity in Provision Desired attitude to environment in textbooks. The First International Conference on Theoretical Foundations of Iranian Visual arts With Environmental Approach. Alzahra University.

[2] Barqarar, M, "A survey on status for specialized manpower in sport entities," Dissertation of Master's Degree progam, Isfahan University.2006.

[3] Zahirinia M., Nikkhah H., Shafiei N, "Investigating the effect of family and mass media on the participation of female students in sports activities. Bi-Quarterly," Journal of Participation and Social Development, vol. 1, no. 1, pp. 4261, 2015. http://dx.doi.org/10.1136/jech.54.9.667.

[4] Safari Shali., The impact, "position of public participation on physical education and girl pupils on sport", congress for determination of position of physical education and girl pupils on sport. Ministry of education of physical education deputy and good health, pp. 33-89, 2002.

[5] Khodadadi, MR., Moharam Zadeh M., Ghorbanzade, B A, "survey on mental hygiene and physical fitness factors, instructor in high school grade, Marand County. The summary of essay of the first specialized congress of physical education and sport science," Education Dept. of western Azerbaijan, 2009. [in Persian].

[6] Ehsani M. "The level of women's sports participation in Iran and the factors hindering sports activities," International Journal of Humanities, no. 3, pp. 15-28, 2005.

[7] Beirami, S, "Compare barriers affecting sports participation of high school students in Isfahan and Urmia," 2008. University of Isfahan. [in Persian].

[8] Moeidfar, S. "Sexual differentiation of villagers in work, leisure, activity and consumption of cultural goods," Iranian Journal of Sociology, vol. 5, no. 2, pp.179-200, 2017.

[9] Farzizadeh Z., Babaei M, "Investigating the leisure situation of women in rural communities," vol. 15, no. 1, pp.101-122, 2017.

[10] Rostami F., Sahraei M., Maleki T., Sasani F., Shahmoradi M, "Pathology Challenges and problems of girls in rural communities," Journal of Women and Policy Development, vol. 12, no. 4, pp. 489-506136, 2014.

[11] Saadi H., Latifi S., Vedadi A, "Investigating the problems of rural girls in Hamadan province," Rural and Development Quarterly, vol. 15, no. 1, pp. 153-176, 2012.

[12] Chen M., Lai L., Chen H., Gaete J, "Development and validation of the short-form adolescent health promotion scale," Bmc Public Health, vol. 14, pp. 91-9, 2014.

[13] Chiou A., Hsu M. Hung, M. "Predictors of health promoting behaviors in Taiwanese patients with coronary artery disease," Applied Nursing Research, no. 30, pp. 1-6, 2016. https://doi.org/10.1016/j.apnr.2015.08.008.

[14] Wilcox S., Castro C., King AC., Housemann R., Brownson R. $\mathrm{C}$, "Determinants of leisure time physical activity in rural compared with urban older and ethnically diverse women in the United States," Journal of Epidemiology \& Community Health, vol. 54, no. 9, pp. 667-672, 2000. http://dx.doi.org/10.1136/jech.54.9.667.

[15] Barnidge, E. K., Radvanyi, C., Duggan, K., Motton, F., Wiggs, I., Baker, E. A., \& Brownson, R. C. "Understanding and addressing barriers to implementation of environmental and policy interventions to support physical activity and healthy eating in rural communities," The Journal of Rural Health, vol. 29, no. 1, pp. 97-105, 2013. https://doi.org/10.1111/j.1748-0361.2012.00431.x.

[16] Mirghafouri SH., Sayadi Toranlu H., Mirfakhrodini SH, "A survey and analyze of the effective issues and problems on women participation in sport activities case study: girl student of Yazd university," Sport management, pp. 83-100, 2009.

[17] Tripathy JP., Thakur JS., Jeet G., Chawla S., Jain S, "Urban rural differences in diet, physical activity and obesity in India: are we witnessing the great Indian equalization? Results from a cross-sectional STEPS survey," BMC Public Health, vol. 16, no. 1, pp. 810-816, 2016.

[18] Deborah A, W, .Charles, AB.," Foundations of physical education, exercise science, and sport". Boston, McGraw-Hill, 15th Ed, 2006.

[19] Laker A, "The Sociology of Sport and physical education, "Rutledge, 1st ed, 2002.

[20] Olsen J M, "An integrative review of literature on the 
determinants of physical activity among rural women," Public Health Nursing, vol. 30, no. 4, pp. 288-311, 2013. https://doi.org/10.1111/phn.12023.

[21] Bantham A., Ross SE T., Sebastião, E., \& Hall, G. "Overcoming barriers to physical activity in underserved populations," Progress in Cardiovascular Diseases, 2020. https://doi.org/10.1016/j.pcad.2020.11.002.

[22] Rimmer JH., Hsieh K., Graham BC., Gerber BS, "Barrier removal in increasing physical activity levels in obese African American women with disabilities," Journal of women's Health, vol. 19, no. 10, pp. 1869-1876, 2010. https://doi.org/10.1089/jwh.2010.1941.

[23] Naqdi A., Balali A., Imani P, "Socio-Cultural barriers to women's participation in sports activities," Women in Development and Politics (women's Research), vol. 9, no. 1, pp. 147-163, 2011.

[24] Eslami Marzanklateh MM., Mousavi SJ., Khoshfar Gh, "Relationship between social capital and women's participation in sports activities," Social Capital Management, vol. 1, no. 2, pp. 139-159, 2014. 10.22059/JSCM.2014.53459.

[25] Riahi MA, "Social factors affecting the acceptance of gender stereotypes," Journal of Women in Development and Politics (women's Research), vol. 1, no. 17, pp. 109-136, 2007.

[26] Tondnavis F, "The place of sports in the leisure time of Iranian women," Journal of Harakat, no. 12, pp. 2002.

[27] Trigueros R., Alias A., Gallardo A M., García-Tascón M., Aguilar-Parra, J. M, "Validation and Adaptation of the Prosocial and Antisocial Behavior in Sport Scale to the Spanish context of Physical Education. International," Journal of Environmental Research and Public Health, vol.17, no. 2, pp. 471-477, 2020. https://doi.org/10.3390/ijerph1702 0477

[28] Bruner M. W., Boardley I D., Benson A J., Wilson K S., Root Z., Turnnidge J., Côté J, "Disentangling the relations between social identity and prosocial and antisocial behavior in competitive youth sport," 2018. Journal of youth and adolescence, vol. 47, no. 5, pp. 113-127, 2013.

[29] Arzu D., Emine H., Leven E. "Perceived barriers to physical activity in university students," Journal of Sport Science and Medicine, vol. no. 49, pp. 46-52, 2006.

[30] Agergaard S, "Religious culture as a barrier? A counter-narrative of Danish Muslim girl's participation in sports," Qualitative Research in sport, Exercise and Health, vol. 8, no. 2, pp. 213-224, 2016.

[31]Foster S., Giles-Corti B, "The built environment, neighborhood crime and constrained physical activity: an exploration of inconsistent findings," Preventive medicine, vol. 47, no. 3, pp. 241-2515, 2008.

[32] Doyle S, "Kelly-Schwartz, A., Schlossberg, M., \& Stockard, J, "Active community environments and health: the relationship of walkable and safe communities to individual health," Journal of the American Planning Association, vol. 72, no. 1, pp. 19-31, 2006. https://doi.org/10.1080/0194436 0608976721 .

[33] Massanori O, "Effect of the physical activity in leisure time and commuting to work on mental health," Journal of Occupational Health, no. 49, pp. 46-52, 2007. https://doi.org/10.1539/joh.49.46.

[34] Saraie S., Amini A., Yousefi A., Fattahi F, "The place of sports in the life of rural women in Isfahan and its deterrents," Bi-Quarterly Journal of Social Participation and Development, vol. 3, no. 5, pp. 27-46, 2017.

[35] Osuji T., Lovegreen S., Elliott M., Brownson R, "Brriers to Physical activity among women in the rural Midwest. Women \& Health," vol. 44, no. 1, pp. 41-55, 2006. https://doi.org/10.1300/J013v44n01_03.

[36] Sit C, Kerr J., Wong I, "Motives for and barriers to physical activity participation in middle-aged Chinese women, "Psychology of Sport and exercise, vol. 9, no.3, pp. 266-283, 2008.https://doi.org/10.1016/j.psychsport.2007.04.0062016.

[37]Zimmermann K., Leslie R., Carnahan Nadine RP, "Age-Associated Perception of Physical Activity Facilitators and Barriers among Women in Rural Southernmost Illinois," Preventing chronic disease, no. 13.

[38] Dagkas S, "Is social inclusion through PE, Sport and PA still a rhetoric?" Evaluating the relationship between physical education, sport and social inclusion," Educational Review, vol. 70, no. 1, pp. 67-74, 2018. https://doi.org/10.1080/0013 1911.2018.1399629.

[39] Evenson K R., Sarmiento O L., Tawney K W., Macon M L., Ammerman, A. S, "Personal, social, and environmental correlates of physical activity in North Carolina Latina immigrants," American journal of preventive medicine, vol. 25, no. 3, pp. 77-85, 2003. https://doi.org/10.1016/S0749-3 797 (03)00168-5.

[40] Torkfar A., Shirazinejad R., Jahromi NN, "Women's Participation in Sports Predictions based on Social, Cultural, Personal, Family Factors, and Facilities in the City Pasargad," Jurnal Fikrah, vol. 8, no. 2, pp. 181-195, 2017.

[41] Nowruzi A., Maleki A., Parsamehr M., Ghasemi H, "Investigating the role of social support and sports consumption in explaining sports participation (a study on women in Ilam province)," Iranian Journal of social studies, no. 2, pp. 241-251, 2017.

[42] Walia S., Liepert B, "Perceived facilitators and barriers to physical activity for rural youth: an exploratory study using photovoice," Rural and Remote Health, vol. 12, no.1842 17, 2012.

[43] Roozbehani M., Kamkari K., Mirzapour A, "Investigating the effective factors on barriers to participation of elderly women in recreational sports activities in Alborz province," Studies of the basics of management in sports, no. 2, pp. 33 42, 2014. 